Kraydashenko O. V., Tyaglaya O. S. Prognostic value of ST2 biomarkers in hypertonic disease patients on the background of the chronic obstructive pulmonary disease. Journal of Education, Health and Sport. 2021;11(09):806-815. eISSN 2391-8306. DOI http://dx.doi.org/10.12775/JEHS.2021.11.09.096

https://apcz.umk.pl/JEHS/article/view/JEHS.2021.11.09.096

https://zenodo.org/record/5585369

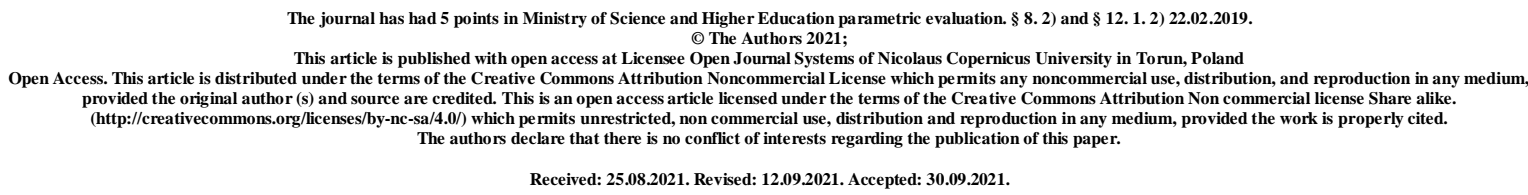

UDC: 616.12-008.331.1: 616.24-007.63

\title{
PROGNOSTIC VALUE OF ST2 BIOMARKERS IN HYPERTONIC DISEASE PATIENTS ON THE BACKGROUND OF THE CHRONIC OBSTRUCTIVE PULMONARY DISEASE
}

\author{
O. V. Kraydashenko ${ }^{1}$, O.S. Tyaglaya ${ }^{2}$ \\ Zaporizhzhya State Medical University, Zaporozhye, Ukraine \\ ${ }^{1}$ Department of Clinical Pharmacology, Pharmacy, Pharmacotherapy and Cosmetology \\ ${ }^{2}$ Department of Internal Diseases
}

\begin{abstract}
Introduction. Scientific research by domestic and foreign scientists and many years of clinical experience suggests that in patients with chronic obstructive pulmonary disease (COPD) a combination with arterial hypertension (AH) were often observed. Soluble form of ST2 protein of the patients is involved in certain inflammatory diseases and in the paracrine system of protection of the heart and lungs as marker, that determines the prognosis in patients with heart and lung deficiency and predicts the death of the patient during the year.
\end{abstract}

The purpose of this study was to evaluate the expression of ST2 protein in patients with arterial hypertension on the background of chronic obstructive pulmonary disease.

Materials and methods. In 23 patients were diagnosed arterial hypertension stage II and COPD stage II without clinically significant concomitant disease, with an average age $51.72 \pm 1.22$ years (49.33-54.09) (gender composition: 22 males and 1 female), the smoking status is comparable to COPD patients, 18 patients with AH of both sexes aged from 33 to 67 
years (mean age $50.74 \pm 1.49$ years (47.81-53.76), male / female ratio 17 / 83\%), stage II of the arterial hypertension with the level of I-III degree of hypertension, different cardiovascular risk, without adequate systematic antihypertensive therapy and 18 patients with COPD stage II, mean age $50.32 \pm 0.99$ years (48.22-52.16) (gender composition: 14 males and 4 females), duration of the disease $7.52 \pm 1.14$ years. $80 \%$ of active smokers, the index of bacon-years $17.23 \pm 2.69$ years, the harmful professional factors (industrial) indicated $23.53 \%$. Participants expressed their willingness to be included in medical research.

Research results. The obtained data indicate that the lowest level of expression of ST2 protein was detected in patients with hypertension without concomitant pathology $21.05 \pm 2.12 \mathrm{ng} / \mathrm{mL}$, which is in $11.78 \%$ lower than in patients with COPD without concomitant pathology $(23,53 \pm 1.8 \mathrm{ng} / \mathrm{mL})$. The highest expression of ST2 protein were demonstrated in patients with comorbid pathology group with COPD on the background of $\mathrm{AH}-33.01 \pm 6.25 \mathrm{ng} / \mathrm{mL}$, which is in $56.82 \%$ higher compared with patients with $\mathrm{AH}$, and in 40.29\% higher than analogous marker in patients with monopathology in the form of COPD.

In the group of COPD patients with a high level of ST2 (more than $30 \mathrm{ng} / \mathrm{ml}$ ), significantly more negatively controlled negative cardiovascular predictors, such as the presence of left ventricular hypertrophy $(\chi 2=7.61$ at $p=0.006)$ and sympathetic balance disturbances according to the LF / HF index $(\chi 2=4.72$ at $p=0.03)$ and ST2 elevation was reliably associated not only with extrapulmonary prognostic factors, but also with a decrease in FEV1 of less than $50 \%(\chi 2=5.45$ at $p=0.02)$.

Conclusions. Patients of the experimental group with comorbid pathology $\mathrm{AH}$ and COPD had the most significant increasing level of ST2 protein as unfavorable prognosis marker compared to the groups of patients without combination of this pathology.

Key words: prognosis; arterial hypertension; chronic obstructive pulmonary disease; soluble form of ST2 protein; cardioprotection.

Introduction. Scientific research by domestic and foreign scientists and many years of clinical experience suggests that in patients with chronic obstructive pulmonary disease (COPD) a combination with arterial hypertension (AH) were often observed. It is known that both COPD and hypertension are multifactorial diseases and develop due to the complex interaction of genetic factors and environmental factors $[1,2]$. In recent years, there has been a significant increase in the number of patients with this comorbid pathology, including Ukraine. COPD in combination with hypertension is a very important medical and social 
problem, the prevalence of this comorbid pathology is on average 34\%, ranging from 17 to $70 \%$, and according to autopsy - up to $85 \%$. The development of disability as a result of these diseases lead to significant direct and indirect economic costs, which places a serious burden on the budget of society and health authorities, so the relevance of the study of this issue is beyond doubt. In the modern literature there are many works devoted to the study of the combined pathology of COPD and hypertension, but some data are still debatable and cause some contradictions $[3,4]$.

A promising biomarker in diseases of the cardiovascular system is stimulating growth factor (ST2). This protein is a member of the interleukin-1 receptor family, which exists in transmembrane form on cardiomyocytes and in soluble form (sST2) in serum. Increased sST2 concentrations are closely associated with the severity of cardiovascular disease, an increased risk of death, and the risk of sudden death in patients, regardless of the volume of the ejection fraction. However, the degree of increase in its concentration in the blood varies greatly, which complicates the use of this marker [5].

Recently published articles describe current ideas about the relationship of ST2 to inflammatory diseases [6]. ST2 is a member of the interleukin-1 receptor family having transmembrane and soluble (sST2 or soluble ST2) isoforms. Concentrations of soluble human ST2 in the circulatory system are elevated in patients with various disorders associated with an abnormal response of T-helper cells type 2 (Th2), including systemic lupus erythematosus and asthma, as well as inflammatory processes that are largely independent of $\mathrm{Th} 2$ responses such as septic shock and trauma. Moreover, the interleukin-33 / ST2L alarm system is a key mechanism of heart protection in case of mechanical overload. Increased soluble form of ST2 in humans is also a marker of poor prognosis in patients with heart failure and myocardial infarction [7]. Elevated levels of soluble human ST2 are also a prognostic factor in an individual's death within one year. Soluble form of ST2 protein of the patients is involved in certain inflammatory diseases and in the paracrine system of protection of the heart and lungs as marker, that determines the prognosis in patients with heart and lung deficiency and predicts the death of the patient during the year.

The publication is a fragment of the planned research work of the Department of Clinical Pharmacology, Pharmacy, Pharmacotherapy and Cosmetology of Zaporozhye State Medical University "Clinical-biochemical and morphological aspects of diagnosis and possibilities of pharmacotherapeutic correction of cardiovascular pathology in people of different ages" (state registration № 0114U001393). 
The purpose of this study was to evaluate the expression of ST2 protein in patients with arterial hypertension on the background of chronic obstructive pulmonary disease.

Materials and methods. In 23 patients were diagnosed arterial hypertension stage II and COPD stage II without clinically significant concomitant disease, with an average age $51.72 \pm 1.22$ years (49.33-54.09) (gender composition: 22 males and 1 female), the smoking status is comparable to COPD patients, 18 patients with AH of both sexes aged from 33 to 67 years (mean age $50.74 \pm 1.49$ years (47.81-53.76), male / female ratio 17 / 83\%), stage II of the arterial hypertension with the level of I-III degree of hypertension, different cardiovascular risk, without adequate systematic antihypertensive therapy and 18 patients with COPD stage II, mean age $50.32 \pm 0.99$ years (48.22-52.16) (gender composition: 14 males and 4 females), duration of the disease $7.52 \pm 1.14$ years. $80 \%$ of patients were active smokers, the index of bacon-years is $17.23 \pm 2.69$ years, the harmful professional factors (industrial) were indicated $23.53 \%$. Participants expressed their willingness to be included in medical research.

The diagnosis of COPD was made on the basis of generally accepted criteria. Patients who participated in the study had mostly moderate to severe stage of COPD (GOLD).

The level of forced expiratory volume in first second (FEV1) and the ratio of the volume of forced expiratory volume in first second to the forced vital capacity of the lungs (FEV1 / FVC) were evaluated. Determination of the function of external respiration was performed according to the standard method on a Computer spirography by "SpiroCom" (HAI-Medica, Ukraine) using software in accordance with the recommendations of the American Thoracic and European Respiratory Community. All patients gave written voluntary consent to participate in the study and received traditional therapy, according to WHO recommendations.

The content of ST2 in blood plasma was determined using appropriate ELISA test systems (manufacturer - Bender Medsystems, Austria) in the Training Medical and Laboratory Center of Zaporozhye State Medical University according to the instructions attached to the kit (Head is Prof. Abramov A.V.).

For the statistical data processing we have been used the licensed program "STATISTICA® for Windows 6.1" (StatSoft Inc., USA, serial number RGXR412D674002FWC7). Distribution normality analysis was evaluated by Shapiro-Wilk (W) test. Data are presented as mean and standard error of representativeness of the sample mean $(95 \%$ confidence interval $(95 \% \mathrm{CI}))$. Comparison of groups on a qualitative basis, as well as in the study of the frequency of meeting indicators was performed using the criterion 
$\chi^{2}$ with the analysis of conjugation tables. Differences at a significance level of less than 0.05 were considered significant.

Research results. In the course of the study, we obtained the following values of the level of ST2 protein expression in groups of patients (table).

Table

The level of ST2 protein expression in the groups of subjects

\begin{tabular}{|c|c|c|c|c|c|c|}
\hline Indicator & $\begin{array}{c}\text { Patients } \\
\text { with arterial } \\
\text { hypertension }\end{array}$ & $\begin{array}{c}\text { Patients with } \\
\text { COPD }\end{array}$ & $\begin{array}{c}\text { Patients with } \\
\text { arterial } \\
\text { hypertension } \\
\text { and COPD }\end{array}$ & \multicolumn{4}{|c|}{$\begin{array}{c}\text { The magnitude of the } \\
\text { differences }\end{array}$} \\
\cline { 2 - 7 } & 1 & 2 & 3 & 2 vs. 1 & 3 vs. 1 & 3 vs. 2 \\
\hline ST2, ng / ml & $21,05 \pm 2,12$ & $\begin{array}{c}23,53 \pm 1,8 \\
(20,01- \\
27,05)\end{array}$ & $\begin{array}{c}33,01 \pm 6,25 \\
(20,76- \\
45,26)^{*}, \#\end{array}$ & $11,78 \%$ & $56,82 \%$ & $40,29 \%$ \\
\hline
\end{tabular}

Note: $*$ - $\mathrm{p}<0.05$ when compared with a group of patients with arterial hypertension; \# $-\mathrm{p}<0.05$ when compared with a group of patients with COPD.

The obtained data indicate that the lowest level of ST2 protein expression was found in patients with hypertension without concomitant pathology $-21.05 \pm 2.12 \mathrm{ng} / \mathrm{ml}$, which is $11.78 \%$ lower than in patients with COPD without concomitant pathology $(23.53 \pm 1.8 \mathrm{ng} /$ $\mathrm{ml})$. The highest values of ST2 protein were shown in patients of the comorbid nosology group with COPD on the background of hypertension $-33.01 \pm 6.25 \mathrm{ng} / \mathrm{ml}$, which is $56.82 \%$ more than in patients with hypertension, and $40.29 \%$ more than same marker in patients with monopathology in the form of COPD.

Thus, the compensatory possibility of the patient's body, which is to include a cardioprotective signaling cascade to prevent fibrosis and remodeling the heart in the most optimal way will be realized in patients with hypertension, to a lesser extent in patients with COPD. The presence of comorbid pathology leads to overexpression of gene 2 and overproduction of ST2 protein, which inhibits the production of interleukin-33 and the inclusion of cardioprotective mechanisms, and indicates an increased risk of adverse prognosis for this cohort of patients.

Also, in the group of patients with COPD on the background of hypertension with high levels of ST2 (more than $30 \mathrm{ng} / \mathrm{ml}$ ) were significantly more likely to verify such negative cardiovascular predictors as left ventricular hypertrophy $\left(\chi^{2}=7.61\right.$ at $\left.\mathrm{p}=0.006\right)$ and violation of sympatho-vagal balance according to $\operatorname{LF} / \operatorname{HF}(\chi 2=4.72$ at $p=0.03)$, and, 
importantly, ST2 elevation was significantly associated not only with extrapulmonary prognostic factors, but also with a decrease in FEV1 less than $50 \%\left(\chi^{2}=5,45\right.$ at $\left.\mathrm{p}=0.02\right)$.

Discussion. ST2 (Growth stimulating factor expressed by gene 2, also known as IL1RL1) is a member of the interleukin-1 (IL-1) family of receptors. ST2 protein has 2 isoforms directly involved in the development of cardiovascular pathology: soluble form (sST2) and membrane-bound form of the receptor (ST2L). The ST2 ligand is the cytokine Interleukin-33 (IL-33).

Healthy heart tissue response to injury or mechanical stress involves the production and binding of interleukin-33 (IL-33) to ST2L, triggering a cardioprotective signaling cascade to prevent fibrosis, heart remodeling, and heart failure (HF). The sST2 blocks the cardioprotective effect of IL-33.

Interleukin-33 (IL-33) is a ligand for ST2, a member of the Toll-like / interleukin-1 receptor superfamily, which is associated with the accessory protein IL-1RAcP [8]. After activation of ST2 / IL-1RAcP by interleukin-33, the signaling cascade is stimulated by lowerlevel molecules such as MyD88 (myeloid differentiation factor 88) and TRAF6 (tumor necrosis factor receptor binding factor 6), which leads to among other things, to the activation of NFKB (nuclear factor-kB). The IL-33 signaling system is involved in the pathogenesis of various diseases and pathologies.

Elevated concentrations of circulating ST2 in the blood indicate a high risk of adverse effects, hospitalization and even death not only for patients with HF, but also for patients with other forms of cardiovascular disease and in the population as a whole. The average normal concentration of ST2 is $18 \mathrm{ng} / \mathrm{ml}$, a concentration above $35 \mathrm{ng} / \mathrm{ml}$ indicates an increased risk. Determination of ST2 in patients with HF may reduce the risk of re-hospitalization and sudden death. For patients with a value of ST2 $>=35 \mathrm{ng} / \mathrm{ml}$, special treatment is prescribed, which includes monitoring the patient with the appointment of additional examinations and diagnostic procedures, medications.

A number of studies have used a method that further determines the amount of interleukin-1 (ST2) receptor 4, according to which COPD is diagnosed when the amount of ST2 is increased compared to the amount of ST2 in healthy people, or according to which the risk of COPD is diagnosed when ST2 is reduced compared to the amount of ST2 in healthy people. The amount of interleukin-1 (ST2) receptor 4 in blood samples from a healthy person is in the range preferably from 50 to $150 \mathrm{pg} / \mathrm{ml}$, preferably from 60 to $140 \mathrm{pg} / \mathrm{ml}$, more preferably from 70 to $130 \mathrm{pg} / \mathrm{ml}$. 
Scientific research [9] of outpatients with chronic heart failure (CHF) have been observed for a long time. The work of these authors was the largest study that examined the predictive significance of ST2 levels in the stable course of CHF. This study showed the high diagnostic value of ST2 in CHF, as well as the fact that the combined measurement of ST2 and NT-proBNP (N-terminal pro-brain natriuretic peptide) was more informative $(14.9 \%)$ to determine the risk than the isolated determination of these markers. These data are confirmed by a study of another authors, which aimed to determine the relationship between ST2 levels and the structural and functional state of the myocardium [10]. In this work, it was confirmed that the combination of elevated concentrations of ST2 and BNP had a greater prognostic value compared to the corresponding indicator in the case of increased activity of only one marker. Thus, in patients who had a simultaneous increase in the concentration of ST2 and BNP, the risk of death during the year (11\%) was 2.6 and 5.5 times higher, respectively, than in persons with increased activity of only one of these markers $(5 \%)$ or with normal values of these peptides $(2 \%)$.

In the following study, the authors examined the underlying mechanisms of rhinovirus(RV)-induced persistent inflammation and progression of emphysema in mice with the COPD phenotype. The results show that 14 days after RV infection, in addition to a steady increase in the expression of CCL3, CXCL-10 and IFN- $\gamma$, as previously observed, the levels of IL-33, ligand for ST2 receptors and matrix metalloproteinases (MMP) 12 are also increased in mice with the COPD phenotype, but not in normal mice. In addition, MMP12 is primarily expressed in CD11b + / CD11c + macrophages. Neutralization of ST2 reduced the expression of CXCL-10 and IFN- $\gamma$ and attenuated the accumulation of CD11b $+/$ CD11c + macrophages, neutrophils and CD8+ T-cells in mice with COPD. Neutralization of IFN- $\gamma$ or ST2 attenuated MMP12 expression and prevented the progression of emphysema in these mice. These results indicate that RV can stimulate the expression of CXCL-10 and IFN- $\gamma$ by activating the ST2 / IL-33 signal axis, which in turn promotes the accumulation of CD11b+ / $\mathrm{CD} 11 \mathrm{c}+$ macrophages and CD8+ T-cells. In addition, RV-induced IFN- $\gamma$ stimulates MMP12 expression, especially in CD11b+/ CD11c+ macrophages, which can destroy alveolar walls, thereby leading to the progression of emphysema in these mice [11].

Biomarkers soluble form ST2 (sST2), galectin-3 and growth and differentiation factor 15 (GDF-15) provide prognostic information in patients with heart failure. The authors of the following study selected 15 patients in each of the following clinical categories: HF without comorbidity, pneumonia without comorbidity, chronic obstructive pulmonary disease (COPD) without comorbidity, HF and comorbidity, kidney disease without comorbidity, sepsis [12]. 
22 healthy people were used as a control group. In each of the 112 study participants, plasma concentrations of sST2 (Presage assay), galectin-3 (Abbott assay), and GDF-15 (Roche assay) were measured.

Compared with the control, the average concentration of sST2 was increased 2.5 times in $\mathrm{CH}, 3.5$ times in pneumonia, 5.0 times in COPD, 5.8 times in $\mathrm{CH}$ and pneumonia, 70 times in sepsis $(\mathrm{p}<0.001$ for all). The sST2 was not significantly increased in renal disease. Elevated plasma concentrations of sST2, galectin-3, and GDF-15 are not specific to a particular disease group, thus the authors conclude that these biomarkers are not suitable for diffiagnostic purposes. What is new in this study is that the indicators of sST2, galectin-3 and GDF-15 as markers of inflammatory diseases are quite sensitive, but not specific enough, which should stimulate further research in this direction.

\section{Conclusions}

1. The presence in the experimental group of comorbid pathology of AH and COPD leads to the most significant elevation of the marker of unfavorable prognosis of ST2 protein compared to groups of patients without a combination of nosologies, which is realized by reducing the activity of cardioprotective signaling cascade and reducing cardioprotective mechanisms.

2. In the group of COPD patients with a high level of ST2 (more than $30 \mathrm{ng} / \mathrm{ml}$ ), significantly more negatively controlled negative cardiovascular predictors, such as the presence of left ventricular hypertrophy $(\chi 2=7.61$ at $p=0.006)$ and sympathetic balance disturbances according to the LF / HF index $(\chi 2=4.72$ at $\mathrm{p}=0.03)$ and ST2 elevation was reliably associated not only with extrapulmonary prognostic factors, but also with a decrease in FEV1 of less than $50 \%(\chi 2=5.45$ at $p=0.02)$.

A promising area for the further study is the effect of differentiated therapy on the level of ST2 protein expression in this cohort of patients with a parallel study of the risk of complications.

\section{References}

1. Jogdand P, Siddhuraj P, Mori M, Sanden C, Jönsson J, Walls AF, Kearley J, Humbles AA, Kolbeck R, Bjermer L, Newbold P, Erjefält JS. Eosinophils, basophils and type 2 immune microenvironments in COPD-affected lung tissue. Eur Respir J. 2020 May 7;55(5):1900110. doi: 10.1183/13993003.00110-2019. PMID: 32060064; PMCID: PMC7236868. 
2. Gimenes JA Jr, Srivastava V, ReddyVari H, Kotnala S, Mishra R, Farazuddin M, Li W, Sajjan US. Rhinovirus-induces progression of lung disease in a mouse model of COPD via IL-33/ST2 signaling axis. Clin Sci (Lond). 2019 Apr 29;133(8):983-996. doi: 10.1042/CS20181088. PMID: 30952808.

3. Paplinska-Goryca M, Misiukiewicz-Stepien P, Proboszcz M, Nejman-Gryz P, Gorska K, Krenke R. The Expressions of TSLP, IL-33, and IL-17A in Monocyte Derived Dendritic Cells from Asthma and COPD Patients are Related to Epithelial-Macrophage Interactions. Cells. 2020 Aug 22;9(9):1944. doi: 10.3390/cells9091944. PMID: 32842623; PMCID: PMC7565129.

4. Tworek D, Majewski S, Szewczyk K, Kiszałkiewicz J, Kurmanowska Z, Górski P, Brzeziańska-Lasota E, Kuna P, Antczak A. The association between airway eosinophilic inflammation and IL-33 in stable non-atopic COPD. Respir Res. 2018 Jun 1;19(1):108. doi: 10.1186/s12931-018-0807-y. PMID: 29859068; PMCID: PMC5984757.

5. Jónsdóttir B, Ziebell Severinsen M, von Wowern F, San Miguel C, Goetze JP, Melander O. ST2 Predicts Mortality In Patients With Acute Hypercapnic Respiratory Failure Treated With Noninvasive Positive Pressure Ventilation. Int J Chron Obstruct Pulmon Dis. 2019 Oct 23;14:2385-2393. doi: 10.2147/COPD.S211448. PMID: 31695358; PMCID: PMC6815753.

6. Huang Q, Li CD, Yang YR, Qin XF, Wang JJ, Zhang X, Du XN, Yang X, Wang Y, Li L, Mu M, Lv Z, Cui Y, Huang K, Corrigan CJ, Wang W, Ying S. Role of the IL33/ST2 axis in cigarette smoke-induced airways remodelling in chronic obstructive pulmonary disease. Thorax. 2021 Feb 15:thoraxjnl-2020-214712. doi: 10.1136/thoraxjnl2020-214712. Epub ahead of print. PMID: 33589512.

7. Teufelberger AR, Nordengrün M, Braun H, Maes T, De Grove K, Holtappels G, O'Brien C, Provoost S, Hammad H, Gonçalves A, Beyaert R, Declercq W, Vandenabeele P, Krysko DV, Bröker BM, Bachert C, Krysko O. The IL-33/ST2 axis is crucial in type 2 airway responses induced by Staphylococcus aureus-derived serine protease-like protein D. J Allergy Clin Immunol. 2018 Feb;141(2):549-559.e7. doi: 10.1016/j.jaci.2017.05.004. Epub 2017 May 19. PMID: 28532656.

8. Saikumar Jayalatha AK, Hesse L, Ketelaar ME, Koppelman GH, Nawijn MC. The central role of IL-33/IL-1RL1 pathway in asthma: From pathogenesis to intervention. Pharmacol Ther. 2021 Sep;225:107847. doi: 10.1016/j.pharmthera.2021.107847. Epub 2021 Apr 2. PMID: 33819560. 
9. Nortamo S, Ukkola O, Lepojärvi S, Kenttä T, Kiviniemi A, Junttila J, Huikuri H, Perkiömäki J. Association of sST2 and hs-CRP levels with new-onset atrial fibrillation in coronary artery disease. Int J Cardiol. 2017 Dec 1;248:173-178. doi: 10.1016/j.ijcard.2017.07.022. PMID: 28942872.

10. Smith D, Helgason H, Sulem P. A rare IL33 loss-of-function mutation reduces blood eosinophil counts and protects from asthma. PLoS Genet. 2017 Mar 8;13(3):e1006659. doi: 10.1371/journal.pgen.1006659. PMID: 28273074; PMCID: PMC5362243.

11. Portelli MA, Dijk FN, Ketelaar ME. Phenotypic and functional translation of IL1RL1 locus polymorphisms in lung tissue and asthmatic airway epithelium. JCI Insight. 2020 Apr 23;5(8):e132446. doi: 10.1172/jci.insight.132446. PMID: 32324168; PMCID: PMC7205441.

12. Kim SW, Rhee CK, Kim KU, Lee SH, Hwang HG, Kim YI, Kim DK, Lee SD, Oh YM, Yoon HK. Factors associated with plasma IL-33 levels in patients with chronic obstructive pulmonary disease. Int J Chron Obstruct Pulmon Dis. 2017 Jan 21;12:395-402. doi: 10.2147/COPD.S120445. PMID: 28176939; PMCID: PMC5268328. 